\title{
Análise da sintomatologia depressiva nos moradores do Abrigo Cristo Redentor através da aplicação da Escala de Depressão Geriátrica (EDG)
}

\author{
Analysis of depression in elderly living in the shelter "Christ the \\ Redeemer", applying the Scale of Geriatric Depression (SGD)
}

Gisela Rocha de Siqueira ${ }^{1}$

Diego Tenório de Vasconcelos ${ }^{1}$

Gustavo Coelho Duarte ${ }^{1}$

Ivo Calado deArruda ${ }^{1}$

João Alysson Silva da Costa ${ }^{1}$

Renata de OliveiraCardoso ${ }^{1}$

${ }^{1}$ Curso de Fisioterapia,

FaculdadeIntegrada do

Recife. Av. Engenheiro

Abdias de Carvalho 1678,

Madalena. 50720-635

Recife PE.

giselarsiqueira@hotmail.com

Abstract The objective of this study was to determine the prevalence of depression in elderly living in the shelter "Christ the Redeemer", in Jaboatão dos Guararapes, Pernambuco. A descriptive study was conducted in September and O ctober 2006, with individuals aged 60 years or more. The sample consisted of 55 elderly, who answered the 30 questions of the Scale of Geriatric Depression of Yesavage. Analysis of variance of the prevalence of depression symptoms between both sexes was conducted. Depression was identified in 28 individuals (51\%), 18 males $(64,2 \%)$ and 10 females $(35,7 \%)$. We recommend the creation of a new scale, simpler and easier to understand for patients with lower cognitive level. Key words Depression, Aged, Scale of Geriatric Depression (SGD)
Resumo 0 objetivo do estudo foi determinar a prevalência de depressão em idososqueresidem no Abrigo Cristo Redentor, Jaboatão dos Guararapes, Pernambuco. Foi realizado estudo descritivo no período de setembro e outubro de 2006, com idosos de idade igual ou acima de 60 anos. A amostra foi composta de 55 idosos, que responderam à Escala de D epressão Geriátrica de Yesavage, com trinta perguntas. Foram realizadas análises de variância da prevalência dos sintomas de depressão entre ambos os sexos. A depressão foi identificada em 28 idosos (51\%), dentre os quais dezoito homens $(64,3 \%)$ e dez mulheres (35,7\%). Recomenda-se a criação de uma nova escala, maissimples ede entendimento maisfácil, para pacientes que têm nível cognitivo rebaixado. Palavras-chave Depressão, I doso, Escala deD epressão Geriátrica (EDG) 
Introdução

Depressão éum problema de saúde freqüenteentre idosos, embora a identificação desses pacientes seja muitas vezes difícil na prática clínica. N esse sentido, a avaliação sistemática dos indivíduos nessa faixa etária pode contribuir para melhorar a detecção dos casos de depressãoํ. 0 estudo do envelhecimento e da vel hice, como processos do ciclo vital, é hojeum dos principais pontos deaten ção dos agentes sociais e governamentais, bem como da medicina em geral. Dentre os diversos transtornos que afetam idosos, a depressão, considerada atualmente o "mal do século", merece especial atenção².

D eacordo com a Organização M undial deSaúde (OM S), desde a década de 1990, a depressão vem ocupando uma posição de destaque no rol dos problemas de saúde pública, considerada a doença mais cara de todas as doenças em todo 0 mundo, e que até 0 ano de 2010 só perderá o primeiro lugar para as doenças cardíacas isquêmicas graves. Ainda segundo a OM S, esta síndrome, no ano de 2020, será a segunda moléstia que afetará os países desenvolvidos e a primeira em países em desenvolvimento ${ }^{3}$.

Depressão écondição clínica freqüente no idoso. Estudos epidemiológicos indicam taxas de pre valência que variam de 1 a 16\% entre idosos vivendo na comunidade. Em indivíduos portadores de doenças clínicas, essas taxas são ainda mais elevadas. Por exemplo, até $45 \%$ dos pacientes com doença coronariana apresentam sintomas depressivos graves. Taxas semel hantes são descritas em associação com uma série de doenças clínicas típicas do idoso, como doença de Parkinson, doença cerebrovascular edoença deA Izheimer. Apesar disso, a presença de sintomas depressivos é apenas ocasionalmente reconhecida pelo paciente e profissionais de saúde, causando sofrimento desnecessário àqueles que não recebem tratamento, dificuldades para os familiares do paciente e elevado custo econômico à sociedade.

A depressão é um problema comum em pessoas idosas, especial mente entre as que possuem várias enfermidades médicas. 0 envelhecimento provoca numerosas perdas que poderiam influenciar a produção de uma síndrome depressiva, como, por exemplo, a perda da saúde, do companheiro, dos papéis sociais ${ }^{4}$, bem como 0 abandono, 0 isolamento social, a incapacidade de reengajamento na atividade produtiva, a ausência de retorno social do investimento escolar ea aposentadoria quemina os recursos mínimos de sobrevivência. Esses fatores comprometem a qualidade de vida e predispõem o idoso ao desenvolvimento da depressão ${ }^{5}$.
Os transtornos do humor são uma das desordens psiquiátricas mais comuns em idosos, sendo responsáveis pela perda de autonomia e pelo agravamento de quadros patológicos preexistentes. Dentre eles, a depressão é a mais freqüente e está associada ao maior risco de morbidade e de mortalidade, ao aumento na utilização dos serviços de saúde, à negligência no autocuidado, à adesão reduzida aos regimes terapêutico e maior risco de suicídio ${ }^{6}$, além de ser caracterizada pela falta de controle sobre o próprio estado emocional ${ }^{7}$.

Do ponto de vista epidemiológico, estima-se que cerca de $15 \%$ dos idosos apresentam algum sintoma de depressão, sendo $2 \%$ do tipo grave. Em algumas populações (hospitalizadas ou institucional izadas), a freqüência é mais el evada, atingindo de $5 \%$ a $13 \%$ dos pacientes hospitalizadose de $12 \%$ a $16 \%$ dos residentes em asilos ${ }^{3}$.

A incidência de depressão é mais elevada em populações asilares ou em hospitais para internação de doentes agudos do que na comunidade. As taxas de sintomas depressivos nessas populações são de $31 \%$ e $23 \%$, respectivamente. Cerca de $13 \%$ dos idosos asilados desenvolvem episódio depressivo dentro de um ano ${ }^{3}$.

Os sintomas da depressão são muito variados, indo desde as sensações de tristeza, passando pelos pensamentos negativos atéas al terações da sensação corporal, como dores e enjôos. Contudo, para se fazer o diagnóstico, énecessário um grupo de sintomas centrais, como a perda de energia ou interesse, humor deprimido, dificuldade de concentração, alterações do apetite e do sono, sentimento de pesar ou fracasso elentificação das atividades físicas e mentais ${ }^{8}$.

Os sintomas corporais mais comuns são sensação de desconforto no batimento cardíaco, constipação, dores de cabeça, dificuldades digestivas. Períodos de melhoria e piora são comuns, o que cria a falsa impressão de que se está melhorando sozinho quando durante alguns dias o paciente sente-se bem. Em geral, tudo se passa gradualmente, não necessariamente com todos os sintomas simultâneos; aliás, é difícil ver todos os sintomasjuntos. Até que se faça o diagnóstico, praticamente todas as pessoas possuem explicações para o que está acontecendo com elas, julgando sempre ser um problema passageiro ${ }^{8}$.

Alguns sintomas podem vir associados aos sintomas centrais, por exemplo, o pessimismo, dificuldade de tomar decisões, dificuldade para começar a fazer suas tarefas, irritabilidade ou impaciência, inquietação, achar que não vale a pena viver, desejo de morrer, chorar à toa, dificuldade para chorar, sensação de que nunca vai melhorar, de- 
sesperança, dificuldade de terminar as coisas que começou, sentimento de pena desi mesmo, persistência de pensamentos negativos, queixas freqüentes, sentimentos de culpa injustificáveis, boca ressecada, constipação, perda de peso e apetite, insônia e perda do desejo sexual ${ }^{8}$.

Em pacientes idosos, além dos sintomas comuns, a depressão costuma ser acompanhada com queixas somáticas, hipocondria, baixa auto-estima, sentimento de inutilidade, humor disfórico, ten dência autodepreciativa, alteração do sono e do apetite, ideação paranóidee pensamento recorrente desuicídio.

Deve-se, ainda, registrar que períodos prolongados de dor, comprometimento da nutrição, emagrecimento e fatores oriundos de doenças físicas que conduzem à diminuição da autonomia e perda da mobilidade física contribuem decisivamente para a instalação da depressão. Pacientes nessas condições tendem a evoluir com sintomas psicóticos caracterizados por delírios de nutilidade e de que seu corpo está morrendo. 0 medo da progressão da doença física, da perda da dignidade e o medo de se transformar em sobrecarga aos familiares também são fenômenos psicológicos que acompanham o comprometimento da condição física ${ }^{5}$. Isso torna o diagnóstico dessa condição mais complexo nesta população².

O paciente deprimido diminui o autocuidado, recusa-se a se alimentar e a seguir as recomendações do clínico, permanecendo por maior tempo restrito ao leito ou com pouca mobilidade física. Estes fatores, associados à debilidade clínica geral, podem diminuir a imunidade, com maior vulnerabilidade a processos infecciosos 5 .

É fundamental, portanto, que os profissionais de saúde tenham familiaridade com as características da depressão no idoso e estejam preparados para investigar a presença de sintomas depressivos entre aqueles em contato com eles. N esse sentido, o uso sistemático deescal as de depressão pode facilitar a detecção desses casos na prática clínica. A escolha da escala vai depender de uma série de fatores, como sua capacidade para detectar casos, sua sensibilidade para monitorar mudanças ao Iongo do tempo, a consistência de suas medidas e a facilidade com a qual ela pode ser administrada.

A Escala de Depressão Geriátrica (EDG) é um dos instrumentos mais freqüentemente utilizados para o rastreamento de depressão em idosos. Diversos estudos mostraram que ela oferece medidas válidas e confiáveis. Descrita em língua inglesa por Yesavage et al. ${ }^{9}$, a escal a original tem trinta itens e foi desenvolvida especialmente para o rastreamento dos transtornos de humor em idosos, com perguntas que evitam a esfera das queixas somáticas. Entre as suas vantagens, destacam-se: é composta por perguntas fáceis de serem entendidas, tem pequena variação nas possibilidades de respostas, pode ser auto-aplicada ou aplicada por um entrevistador treinado 6 .

Tendo em vista o quadro apresentado e o grande interesse no tema por parte das ciências gerontológicas, este estudo foi elaborado com o objetivo de investigar, em uma amostra de base populacional, o índice de depressão em idosos de um asilo em Jaboatão dos Guararapes, Pernambuco.

No idoso institucionalizado, ainda são poucos os trabalhos com relação à incidência de depressão; devido a isso, a importância de tal estudo com relação a essa população.

\section{M etodologia}

Foi realizado um estudo descritivo, utilizando-se, como meio, um questionário padronizado. 0 questionário aplicado é denominado Escala de D epressão Geriátrica (EDG), utilizada para avaliar sintomatologia depressiva e tendo como característica ser auto-aplicativo. A Escala deDepressão Geriátrica é composta por trinta perguntas, cada qual com duas alternativas (sim e não) com valores atribuídos de 0 a 1 e o escore foi dado pela somatória do total de alternativas. Foi considerado o escore 0-10 para normal e 11 ou mais para provável depressão.

A população estudada foi de 55 pessoas ( 31 homens e 24 mulheres), correspondendo a $45 \%$ do total de habitantes do local de estudo. Essa população era composta por indivíduos com mais de 60 anos de idade moradores do Abrigo Cristo Redentor no município de Jaboatão dos Guararapes, Pernambuco. 0 estudo foi realizado no período de setembro e outubro de 2006.

A seleção dos indivíduos estudados foi baseada nos seguintes critérios: 1) idade maior ou igual a 60 anos; 2) capacidadefísica emental para responder 0 questionário; 3) aceitação concedida após explicação do objetivo desse estudo. A distribuição dos questionários foi dada a todos os pacientes queestivessem sendo atendidos pelos acadêmicos de fisioterapia do 80 período da Faculdade Integrada do Recife (FIR), nas quartas esextas no turno da tarde.

A Escala de Depressão Geriátrica foi aplicada pelos acadêmicos de fisioterapia do 8o período da FIR. Foi realizado um treinamento com os mesmos, tendo como objetivo evitar a interferência de acompanhantes e fatores ambientais. Os acadê micos da FIR deslocaram-se até as dependências dos indivíduos estudados. 
Foram usados como critérios de inclusão: pacientes de ambos os sexos, com idade igual ou superior a 60 anos e que apresentassem bom nível cognitivo.

Foram usados como critérios de exclusão: pacientes com patologias que iriam interferir na avaliação da escala e que não se predispusessem a responder o questionário.

Todos os pacientes queforam submetidos a esse estudo assinaram um termo de consentimento livre esclarecido, dispondo-se a participar do estudo. Esse termo tem a explicação do objetivo do estudo, bem como a explicação da EDG.

\section{Resultados}

Através da análise dos resultados encontrados na pesquisa, obteve-se um total de 55 idosos, sendo 31 homens (56,36\%) e 24 mulheres ( $43,64 \%$ ).

Com relação à prevalência dos idosos estudados na instituição, verificou-se que 28 deles (51\%) apresentaram escore igual ou maior a 11, caracterizando uma sintomatologia depressiva, como mostra a Figura 1. Os 49\% restantes não foram caracterizados como depressivos, tendo em vista que seu escore variou entre 2 e 10 pontos.

Com relação ao gênero, verificou-se a prevalência no sexo masculino; dezoito homens (64,3\%) contra apenas dez mulheres $(35,7 \%)$, como mostra a Figura 2.

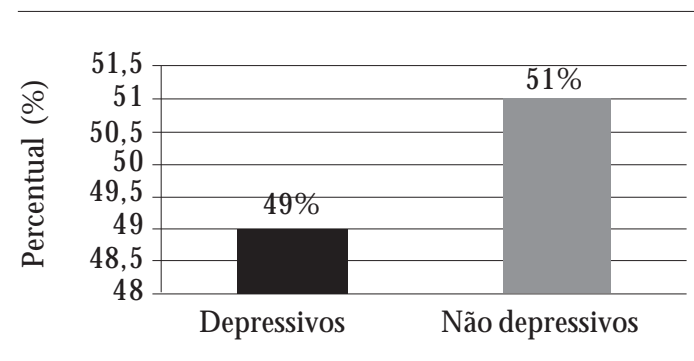

Figura 1. População estudada: depressivos x não depressivos.

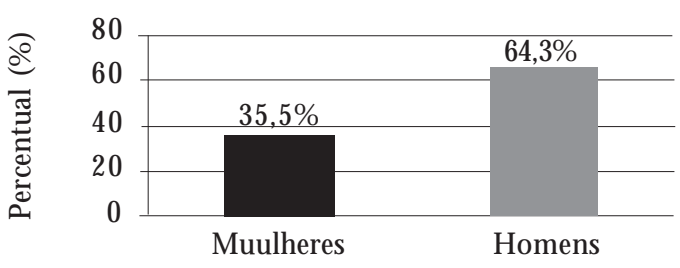

Figura 2. Idosos com depressão: homens x mulheres.
Analisando a média de internamento dos idosos do Abrigo Cristo Redentor, foram encontrados os valores de 11 e 5 anos para mulheres e homens, respectivamente, como mostra a Figura 3.

No quesediz respeito à média deidade da amostra estudada, encontramos 79 e 74 anos para muIheres e homens, respectivamente, como mostra a Figura 4. Analisando os idosos de ambos os sexos, foram encontradas a idade máxima de 100 anos, idade média de 77 anos, idade mínima de 61 anos, com desvio padrão de 10,95.

\section{Discussão}

0 processo de envelhecimento populacional em curso no país tem aumentado a freqüência de doenças psiquiátricas, entreas quais, a depressão, que é a desordem mais comum nesse segmento etário. As taxas de prevalência variam entre $5 \%$ e $35 \%$, quando consideramos as diferentes formas ea gravidade da depressão.

Um aspecto relevanteé que, apesar de boa parte das depressões nos idosos apresentar um quadro clínico semelhante ao de outras faixas etárias, esses indivíduos, com maior freqüência, apresentam quadros atípicos, ou particularidades, como problemas clínicos e sociais simultâneos, que podem levar a dificuldades diagnósticas ${ }^{10}$.

Cheloni et al. ${ }^{11}$ afirmaram que as escalas de depressão no idoso, apesar de úteis no diagnóstico

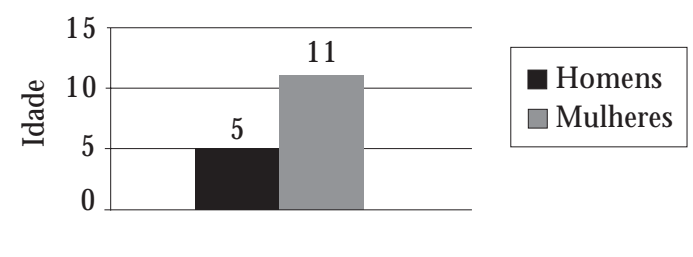

Figura 3. M édia de internamento dos idosos avaliados.

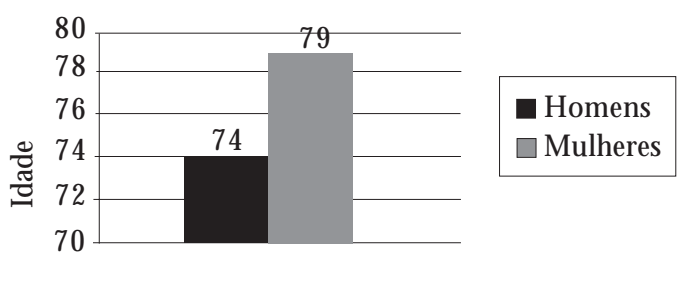

Figura 4. Idade média dos idosos avaliados. 
da depressão, apresentam suas limitações, que devem ser levadas em conta; mas, por outro lado, sua utilização serve de screening para melhor avaliar esses pacientes.

Embora o fato de diversos estudos já terem demonstrado quea EDG oferece medidas válidase confiáveis para a avaliação de transtornos depressivos e da mesma ser bastante utilizada, nos idosos que participaram da nossa pesquisa, este instrumento foi de difícil aplicação por mostrar-se confuso para os mesmos, já que eles têm baixo nível cognitivo.

Nesse estudo, foram identificados $51 \%$ de casos de depressão entre os 55 idosos avaliados. Esse achado é considerado bastante elevado se compararmos aos estudos internacionais realizados em comunidades com idosos, onde também são usadas escalas para diagnosticar depressão. Segundo Lima $^{12}$, essas divergências depercentuais de depressão dos estudos internacionais podem ser atribuídas às diferentes escalas empregadas para diagnosticar depressão, ou a alguns problemas metodológicos, ou, ainda, às possíveis diferenças sociodemográficas observadas em cada país.

0 presente estudo mostra um percentual de depressão nos idosos semel hante aos estudos realizados no Brasil, como o de Cherloni et al. ${ }^{11}$, que através de uma pesquisa sobre a prevalência de depressão nos idosos institucionalizados em M ossoró, Rio Grande do Norte, encontrou um acometimento de $51 \%$ da amostra estudada e, assim como nosso trabalho, esteve compatível com alguns dados encontrados na literatura.

No entanto, esse resultado foi extremamente superior ao encontrado por Aguiar \& Dunningham, citados por Oliveira et al. ${ }^{13}$, que, no Estado da Bahia, encontraram sintomas depressivos em apenas $15 \%$ dos idosos da comunidade. A provável explicação para esse percentual tão alto de idosos deprimidos é que, atualmente, a idéia que se tem sobre a idade avançada é que ela é desprovida de força, incapacidade de prazer, solitária e repleta de amargura.

Antigamente, al gumas sociedades garantiam ao idoso o poder, a honra e o respeito; no entanto, na sociedade moderna, consumista e imediatista, os idosos são encarados como um peso social, semprerecebendo benefício enada oferecendo em troca. Os valores da juventude predominam como os de beleza, de energia e de ativismo, como afirmaram Oliveira et al. ${ }^{13}$.

0 idoso institucionalizado é obrigado a adaptar-se a uma rotina de horários, a dividir seu ambiente com desconhecidos e à distância familiar. $\mathrm{A}$ individualidade e o poder de escolha são substitu- ídos pelo sentimento de ser apenas mais um dentro daquela coletividade ${ }^{11}$. Esses, entre outros, são fatores determinantes para o surgimento de de pressão nos idosos institucionalizados, já que freqüentemente se observa que 0 idoso deprimido passa por uma importantepiora de seu estado geral e por um decréscimo significativo de sua qualidade de vida ${ }^{11}$.

Com relação ao gênero, tivemos uma predominância do sexo masculino, com dezoito homens $(64,2 \%)$ contra dez mulheres (35,7\%). A prevalência da depressão em homens como resultado do nosso estudo ocorreu, provavelmente, devido à região do Nordeste brasileiro apresentar os homens como centro da família e detentores de maior poder e prestígio. À medida que eles vão enveIhecendo, passam a ser vistos como peso para família e começam a se isolar, enquanto que as muIheres possuem uma maior facilidade de relacionamento e companheirismo, por serem educadas, desde crianças, para cuidar da casa e da família.

Os dados do presente estudo não corroboram com os resultados encontrados em alguns trabaIhos da literatura, em que as mulheres são mais acometidas por depressão que os homens, conforme 0 estudo de Almeida ${ }^{1}$. Nesse estudo, Almeida selecionou 64 indivíduos com 60 ou mais anos de idade, atendidos de forma consecutiva nos ambulatórios da Unidade de Idosos do Departamento de Saúde M ental da Santa Casa de São Paulo, entre fevereiro e maio de 1998, e encontrou maior índice de depressão no sexo feminino ( $67,3 \%$ dos casos).

Porcu et al. ${ }^{4}$, em seu estudo sobre a prevalência da sintomatologia depressiva na população de idosos com idade superior a 60 anos, numa área adstrita do PSF, no município de Maringá, Estado do Paraná, encontraram, com relação à sintomatologia depressiva, o escore de $48 \%$ em sua amostra. 0 humor disfórico foi de $16,2 \%$ do total da amostra, sendo que se distribuiu entre $15,1 \%$ dos homens e $17,2 \%$ das mulheres, prevalecendo o feminino. Já o humor depressivo foi de $35,8 \%$ do total da amostra, que se distribuiu em $34 \%$ nos homens e $37,7 \%$ nas mulheres; mais uma vez, o sexo feminino prevaleceu.

E ainda, Linhares et al. ${ }^{14}$, em seu trabalho realizado no ambulatório de um hospital do estado do Distrito Federal, constataram que, dos setenta homens submetidos à EDG versão reduzida, 55 (78,6\%) obtiveram entre 0 a 5 pontos (ausência de depressão); os quinze idosos restantes (21,4\%) encontram-sena faixa de 6 a 15 pontos, indicativa de depressão. A pontuação média masculina na escala correspondeu a $3,6(d p=3,2)$. No que diz respeito às 148 mulheres que responderam à Escala 
de Depressão Geriátrica, 83 (56\%) estão na faixa de 0 a 5 pontos, e 65 (44,0\%) obtiveram entre 6 a 15 pontos, o que sinaliza a presença de sintomas depressivos. A pontuação média feminina foi de $5,3(d p=3,8)$. I sso comprova, mais uma vez, a maior incidência de sintomatologia depressiva no sexo feminino, o que vai de encontro aos resultados obtidos no nosso estudo.

Ao comparar a idade média e a média de internamento dos idosos do Abrigo Cristo Redentor e entres homens e mulheres, encontra-se maior prevalência de ambas nas pacientes do sexo feminino.

Essas variáveis (idade e tempo de internamento) em geral são diretamente proporcionais ao aparecimento da depressão; porém, esses dados obtidos divergiram do estudo, já que, pelos resultados, as mulheres estariam predispostas a desenvolver depressão, pois essas variáveis apresentaram-se em maiores proporções no sexo feminino do que no sexo masculino.

Esse conflito de resultados pode ter acontecido pelo fato do número de homens entrevistados ter sido maior do que o número de mulheres, pois estas apresentaram baixo nível cognitivo, dificultando na resposta dos questionários aplicados; dessa maneira, participaram do critério de exclusão.

0 atendimento emergencial de idosos deve, também, considerar que a depressão está associada à maior morbidade e mortalidade nessa faixa etária, particularmente quando doença física ou declínio cognitivo estão presentes ${ }^{15}$. Outro importante complicador dos quadros depressivos é o maior risco de suicídio - depressão está claramente presente em pelo menos $50 \%$ dos casos letais de suicídio em idosos ${ }^{16,17}$.

\section{Colaboradores}

GR Siqueira foi responsável pela idealização do artigo e supervisão da redação. DT Vasconcelos e GC Duarteforam responsáveis pela concepção do artigo, pesquisa, metodologia e elaboração dos gráficos. IC Arruda foi responsável pela concepção do artigo, coleta de dados, pesquisa. JAS Costa foi responsável pela concepção do artigo, coleta de dados, revisão da literatura e RO Cardoso, pela concepção do artigo, revisão da literatura, coleta de dados e redação final.

\section{Consideraçõesfinais}

Essa pesquisa sugere que os idosos asilados no Abrigo Cristo Redentor apresentam índices altos desintomatologia depressiva segundo a EDG eque essa prevalência é maior em homens do que em mulheres. Em virtude das dificuldades de aplicação da escala nos idosos, sugere-se, então, a criação deuma nova escala, mais simples ede entendimento mais fácil para pacientes que têm nível cognitivo rebaixado.

É importante ressaltar que apenas a aplicação daEDG não éo suficiente para diagnosticar o quadro depressivo do idoso.

Em toda situação de depressão, a abordagem médica deve ser muito cuidadosa, sendo fundamental um detal hado levantamento de dados pessoais, tipo de medicamentos utilizados e antecedentes de problemas psicológicos, além deum exame clínico completo associado à avaliação psiquiátrica e neurológica, que são indispensáveis.

É importante que os profissionais de saúde estejam cientes das particularidades dos quadros clínicos dos idosos e tenham condições de conduzir deforma adequada o tratamento daqueles quetêm problemas de saúde mental. Daí a importância da fisioterapia no trabal ho com o idoso, para melhorar seu bem-estar, condição física, independência, qualidade de vida e auto-estima, e dessa maneira, diminuir os sintomas de depressão.

Sugere-se também que outros trabalhos sejam realizados com o intuito de se desenvolver programas de prevenção e proteção à saúde de idosos que vivem em abrigos, para evitar essa el evada prevalência de depressão. 


\section{Referências}

1. Almeida OP, Almeida SA. Confiabilidade da versão brasileira da Escala de Depressão em Geriatria (GDS) versão reduzida. Arq N euro-Psiquiatr 1999; 57(2B).

2. Gazalle FK, Hallal PC, Lima MS. Depressão na população idosa: os médicos estão investigando? Rev Bras Psiquiatr 2004; 26(3).

3. Coutinho MPL, Gontiès $B$, Araújo LF, Sá RCN. Depressão, um sofrimento sem fronteira: representações sociais entre crianças e idosos. Psico-USF 2002; 8(2):183-192.

4. Porcu M, Fritzen CV, Cano M FF, Bemvides M N, Colombari I. Prevalência da sintomatologia depressiva em idosos em uma área de saúde da família no município de M aringá, Estado do Paraná. Acta Scientiarum 2002; 24(3):703-705.

5. Stella F, Gobbi S, Corazza DI, Costa JLR. Depressão no idoso: diagnóstico, tratamento e Benefícios da Atividade Física. M otriz 2002; 8(3):91-98.

6. Paradela EM P, Lourenco RA, Veras RP. Validação da escala de depressão geriátrica em um ambulatório geral. Rev. Saúde Pública 2005; 39(6):918-923.

7. Garcia A, Passos A, Compo AT, Pinheiro E, Barroso F, Coutinho G. A depressão e o processo de envelhecimento. Ciências e Cognição 2005; 7:111-121.

8. Marot R. Depressão: transtornos relacionados por semelhança ou classificação. 2004; [acessado 2006 ago 29]. Disponível em: http://www.psicosite.com.br/tra/ hum/depressao.htm

9. Yesavage JÁ, Brink TL, Rose TL, Lum O, Huang V, Adey M, Leirer VOI. Development and validation of a geriatric depression screening scale: a preliminary report. J Psychiat Res 1983; 17(1):37-49.
10. Leite VM M, Carvalho EM F, Barreto KML, Falcão IV. Depressão e envelhecimento: estudo nos participantes do Programa Universidade Aberta à Terceira Idade. Rev Bras Saúde M ater Infant 2006; 6(1):31-38.

11. Cherloni CFP, Pinheiro FLS, Filho MC, Medeiros AL. Prevalência de depressão em idosos institucionalizados no município de Mossoró/RN segundo escala de depressão geriátrica (Yesavage). Expressão 2003; 34(1-2): 61-73.

12. Lima MS. Epidemiologia e impacto social. Rev Bras Psiquiatr 1999; 21:1-5.

13. Oliveira DAAP, Gomes L, Oliveira RF. Prevalência de depressão em idosos que freqüentam centros de convivência. Rev. Saúde Pública 2003; 40(4):734-736.

14. Linhares CRC, Coelho VLD, Guimarães RM, Campos APM, Carvalho NT. Perfil da clientela de um ambulatório de geriatria do Distrito Federal. Psicol Reflex Crit 2003; 16(2):319-326.

15. Cole MG , Bellavance F. The prognosis of depression in old age. Am J Geriatr Psychiatry 1997; 5:4-14.

16. Hepple J, Quinton C. One hundred cases of attempted suicide in the elderly. Br J Psychiatry 1997; 171:42-46.

17. Kennedy GJ. Suicide and depression in late life: critical issues in treatment, research, and public policy. N ew York: John Wiley; 1996.

Artigo apresentado em 20/07/2007

Aprovado em 13/12/2007 